\title{
AKT plays a crucial role in gastric cancer (Review)
}

\author{
TAKAMITSU SASAKI ${ }^{1}$, YUICHI YAMASHITA ${ }^{1}$ and HIROKI KUNIYASU ${ }^{2}$ \\ ${ }^{1}$ Department of Gastroenterological Surgery, Fukuoka University School of Medicine, Fukuoka, Fukuoka 812-8582; \\ ${ }^{2}$ Department of Molecular Pathology, Nara Medical University, Kashihara, Nara 634-8521, Japan
}

Received August 5, 2014; Accepted April 29, 2015

DOI: $10.3892 / \mathrm{ol} .2015 .3260$

\begin{abstract}
The AKT protein is involved in the phosphatidylinositol-3 kinase signaling pathway and is a vital regulator of survival, proliferation and differentiation in various types of cells. Helicobacter pylori infection induces epithelial cell proliferation and oxidative stress in chronic gastritis. These alterations lead to telomere shortening, resulting in the activation of telomerase. AKT, in particular, is activated by $H$. pylori-induced inflammation. AKT then promotes the expression of human telomerase reverse transcriptase, which encodes a catalytic subunit of telomerase, and induces telomerase activity, an essential component of the process of carcinogenesis. AKT activation is increased in gastric mucosa with carcinogenic properties and is associated with the low survival of patients with gastric cancer. The findings of the present study suggest that AKT is pivotal in gastric carcinogenesis and progression.
\end{abstract}

\section{Contents}

1. Introduction

2. AKT activation in the gastric mucosa

3. Telomerase

Correspondence to: Professor Hiroki Kuniyasu, Department of Molecular Pathology, Nara Medical University, 840 Shijo-cho, Kashihara, Nara 634-8521, Japan

E-mail: cooninh@zb4.so-net.ne.jp

Abbreviations: PI3K, phosphatidylinositol-3 kinase; VEGF, vascular endothelial growth factor; pAKT, phosphorylated AKT; iNOS, inducible nitric oxide synthase; PTEN, tensin homolog deleted on chromosome 10; NT, nitrotyrosine; hTERT, human telomerase reverse transcriptase; CG, chronic gastritis without H. pylori; CAG, chronic active gastritis with H. pylori; $\mathrm{CMG}$, chronic metaplastic gastritis without $H$. pylori; CGA, chronic gastritis with atypia without $H$. pylori; SHP2, src homology 2 domain-containing protein tyrosine phosphatase-2; hTR, human telomerase RNA; EMT, epithelial-mesenchymal transition; NO, nitric oxide; ROS, reactive oxide species

Key words: Helicobacter pylori, telomerase, hTERT, chronic gastritis
4. Telomerase RNA

5. Telomere shortening

6. Telomeres and AKT

7. Conclusion

\section{Introduction}

Gastric cancer is the fourth most common cancer worldwide (1). Advances in the molecular analysis of preneoplastic and neoplastic lesions of the stomach have revealed a large number of epigenetic and genetic alterations that determine a multistep process of stomach carcinogenesis (2). Helicobacter pylori infection is inherently responsible for the pathogenesis of intestinal metaplasia, and the epidemiological contribution of the infection to gastric cancer is similar in European and Japanese populations (3). In 1994, the International Agency for Research on Cancer recognized H. pylori as a definite carcinogen in gastric cancer, based on a strong epidemiological association between chronic colonization of $H$. pylori and gastric cancer (4). In addition, a Mongolian gerbil model revealed that the inoculation of $H$. pylori into the stomach is closely associated with the occurrence of chronic gastritis, intestinal metastasis and promotion of adenocarcinoma (5).

AKT is a pivotal regulator of cell survival, proliferation and differentiation, and AKT is also a member of the phosphatidylinositol-3 kinase (PI3K) signaling pathway (6-9). Stimulation of receptor tyrosine kinases or G-proteins results in the activation of PI3K, which in turn activates AKT. AKT phosphorylation is catalyzed by heat shock protein 90 , and the dephosphorylation of AKT is mediated through protein phosphatase 2A (9). Subsequently, AKT regulates signaling through various growth factors and cytokines. Upstream of AKT, activation of insulin-like growth factor-1 receptor, epidermal growth factor receptor and human epidermal growth factor receptor 2, which are important in cancer progression, activates AKT $(6,7)$. Therefore, AKT has been reported as a biomarker that predicts metastasis in human gastrointestinal cancer (8).

Phosphorylation of AKT modulates signals from phosphatase and tensin homolog deleted on chromosome 10 (PTEN) and the mammalian target of rapamycin (mTOR), resulting in diverse effects of AKT on cells (9). AKT1 is recognized as an apoptotic inhibitor that contributes to cancer progression (9). Phosphorylation catalyzed by AKT inactivates B-cell 
lymphoma 2 (Bcl-2) antagonist of cell death, resulting in the dissociation of the promoter from Bcl-2. In addition, AKT activates nuclear factor $\kappa \mathrm{B}$, which results in the upregulation of transcription for numerous survival genes (10). AKT also promotes angiogenesis through the upregulation of vascular endothelial growth factor (VEGF) (11). The AKT-microRNA regulatory network suggests that microRNA-mediated gene regulation interacts with the AKT signal pathway (12). Thus, the expression and activation of AKT promotes tumorigenesis, and AKT is a relevant molecular target for cancer treatment (7).

\section{AKT activation in the gastric mucosa}

To establish the role of oxidative stress and AKT activation in gastric cancer development, the levels of phosphorylated AKT (pAKT), inducible nitric oxide synthase (iNOS), nitrotyrosine (NT) and human telomerase reverse transcriptase (hTERT), which is the catalytic component of telomerase, have previously been examined by ELISA in non-cancerous gastric mucosa and gastric cancers (13). In this previous study, the levels of pAKT were found to be directly associated with the levels of iNOS, NT and hTERT. Inflammation of the gastric mucosa was classified into four categories: Chronic gastritis without $H$. pylori (CG); chronic active gastritis with $H$. pylori (CAG); chronic metaplastic gastritis without H. pylori (CMG); and chronic gastritis with atypia without $H$. pylori (CGA). Increased levels of pAKT, iNOS and NT were found in tissues from CG, CAG, CMG and CGA. hTERT protein expression was detected only in CGA. These previous findings suggest that oxidative stress may be implicated in AKT activation and hTERT induction, and also that the presence of CGA in the mucosa may present a high-risk status for gastric carcinogenesis (13).

$H$. pylori infection is the major cause of chronic persistent inflammation of the gastric mucosa $(5,14)$. In gastric mucosal pathology, the infection induces chronic inflammation and increases the production of reactive oxide species (ROS) (3). $H$. pylori stimulates the proliferation of gastric mucosal cells through type IV secretion of cytotoxin-associated gene A (CagA) followed by CagA phosphorylation by src homology 2 domain-containing protein tyrosine phosphatase-2 (SHP2) $(15,16)$. Cag A activates SHP2 phosphatase, which in turn inhibits signal transducers and activators of transcription-mediated growth-suppressive signaling and also activates extracellular signal-regulated kinase-mediated growth signaling (17). The increased growth activity may enhance the risk of gene alteration $(15,16)$.

$H$. pylori-induced inflammation generates morphological changes, including intestinal metaplasia, which is formed by transdifferentiation of the gastric epithelium to the intestinal phenotype (18). In intestinal metaplasia, the antral or fundic mucosa of the stomach is replaced with mucosa that resembles intestinal mucosa (19) through the ectopic expression of caudal type homeobox 2 (20). Intestinal metaplasia differs from normal mucosa in that cell division is accelerated $(21,22)$. Intestinal metaplasia demonstrates several genetic and epigenetic alterations that are also present in gastric cancer (3). It has been reported that transcripts of mutated adenomatous polyposis coli and abnormal cluster of differentiation 44 are present in intestinal metaplasia and gastric cancer (23). Furthermore, a previous study has identified that microsatellite instability and p53 mutation (24) are detected in $33 \%$ of intestinal metaplasias (25). Thus, it is hypothsized that $H$. pylori induces iNOS and the synthesis of ROS, which activates AKT (Fig. 1).

\section{Telomerase}

The telomere is a repetitive 'TTAGGG' sequence that is present at the ends of eukaryotic chromosomes to maintain and protect the integrity of the chromosomes (26). As cells divide, the telomere is shortened in length. Therefore, the length of the telomere acts as a marker of the division limit for cells or for cell death (27). In stem, cancer and cancer stem cells, the telomere is elongated by the activity of telomerase, a ribonucleoprotein enzyme that enables cells to divide endlessly (28). Thus, through the activation of the enzyme, telomerase is a key enzyme for the induction of immortality and malignant properties in somatic cells. Telomerase activity is detected in $85 \%$ of gastric cancer lesions, regardless of tumor stage and histological types (29). In addition, telomerase activity is detected in $23 \%$ of intestinal metaplasia tissues and $50 \%$ of gastric adenoma tissues, whereas no activity in the corresponding gastric normal mucosa has been reported (29).

\section{Telomerase RNA}

Human telomerase RNA ( $h T R)$, which contains 11 nucleotides complementary to the telomere TTAGGG sequence, is generated to function as the template RNA component of telomerase (30). $h T R$ is expressed in pre-crisis cell lines, non-neoplastic tissues, immortalized cell lines and tumor specimens, but the expression level is not associated with the level of telomerase activity (31). Notably, Blasco et al reported that the initial upregulation of $h T R$ is an early event in carcinogenesis and that telomerase is activated only in end-stage tumors during multi-stage carcinogenesis (32).

The expression of $h T R$ and telomerase activity in gastric cancer and corresponding non-cancerous mucosa tissues has been determined previously (33). In this study, telomerase activity was detected in $88 \%$ of carcinoma tissues. Although tumor specimens and non-cancerous mucosa tissues express various levels of $h T R, 81 \%$ of tumor specimens were found to express $h T R$ at an increased level compared with the corresponding mucosa. Gastric carcinoma cell lines were also found to express $h T R$ at high levels (33). Overall, $35 \%$ of non-cancerous mucosa tissues were found to demonstrate telomerase activity, and all non-cancerous mucosa tissues infected with $H$. pylori contained intestinal metaplasia. The incidence of telomerase expression in the mucosa with moderate intestinal metaplasia was revealed to be significantly increased compared with the incidence in mild intestinal metaplasia, whereas $h T R$ overexpression was detected in mild intestinal metaplasia and moderate intestinal metaplasia. Notably, the degree of metaplasia in $H$. pylori-infected mucosa increased concordantly with the level of $h T R$ expression and telomerase activity. These results indicated that $h T R$ overexpression is caused by H. pylori, which plays a role upstream of telomerase activity as an early event in stomach carcinogenesis (33). 


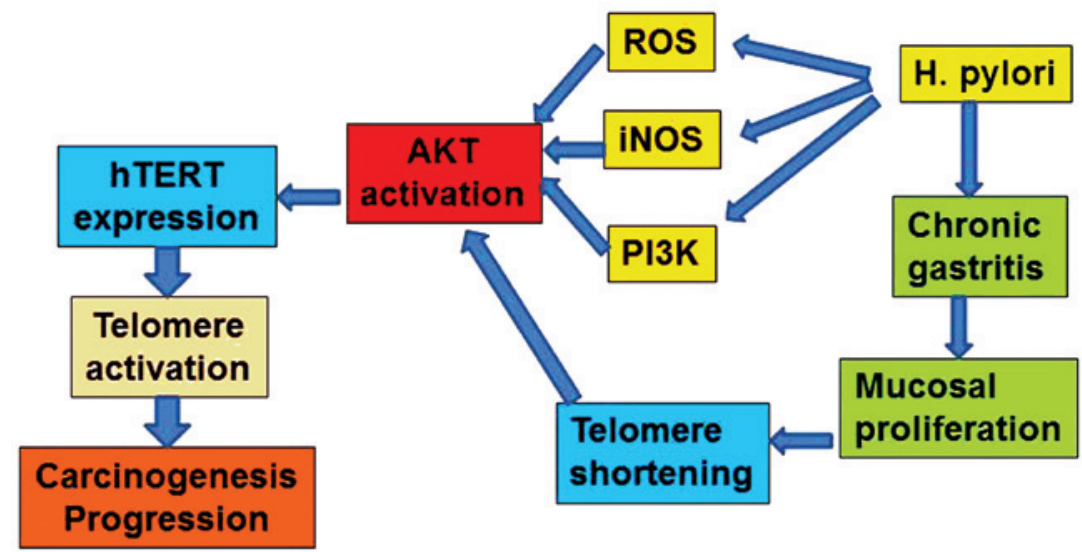

Figure 1. Central role of AKT in H. pylori-induced gastric carcinogenesis. hTERT, human telomerase reverse transcriptase; ROS, reactive oxide species; iNOS, inducible nitric oxide synthase; PI3K, phosphatidylinositol-3 kinase; H. pylori, Helicobacter pylori.

In the stomach, various types of inflammation affect the growth of the mucosa. In particular, intestinal metaplasia and chronic atrophic gastritis exhibit accelerated cell growth and cell cycling compared with $H$. pylori-negative gastric mucosa $(22,34)$. Although the factors that upregulate $h T R$ expression remain unknown, continuous inflammation and regenerative processes may stimulate $h T R$ expression by affecting stem cells, and subsequently, these processes may enhance the activity of telomerase in the non-cancerous mucosa of the stomach (3). Therefore, it is likely that $h T R$ overexpression in non-cancerous mucosa may reflect an earlier process of multistep carcinogenesis in the stomach (33).

\section{Telomere shortening}

The shortening of telomeres in non-cancerous tissues is determined by regenerative processes and is specific to the individual organs or tissue components, since the regeneration of different tissues requires various degrees of cell division, which also occurs in chronic gastritis $(21,22)$. The difference in telomere shortening of the gastric mucosal epithelium has been investigated using an in situ telomere quantification technique (35). The association between telomere length and $H$. pylori infection was assessed, and the difference between the telomere length that characterizes intestinal metaplasia cells in cancer patients and the telomere length in cells obtained from non-cancer patients was also examined (35).

In healthy individuals without $H$. pylori infection, the telomere volumes in gastric epithelial tissues were found to be $70-79 \%$ of the volume of the telomeres in intramucosal lymphocytes, which acted as an internal control (35). In patients without gastric cancer, the telomere volume in $H$.pylori-infected mucosa cells was found to be significantly decreased compared with $H$. pylori-negative mucosa cells in metaplastic and non-metaplastic tissues $(\mathrm{P}<0.0001)(35)$. In gastric cancer patients, the telomere volume in intestinal metaplasia cells adjacent to a cancerous lesion was identified as $75 \%$ of the telomere volume in intestinal metaplasias in patients without cancer $(\mathrm{P}=0.0001)$ (35). Therefore, H. pylori infection is strongly associated with the shortening of telomeres, which subsequently stimulates necessary elongation of the telomeres by telomerase activity, particularly in the gastric epithelium (35).

\section{Telomeres and AKT}

The catalytic subunit of hTERT, which is responsible for telomerase activity and telomere elongation, is suppressed in differentiated cells (36). Shortening of the telomeres has been identified as a significant factor for the induction of $h T E R T$ expression in the gastric mucosa (35). hTERT expression is detected in $7 \%$ of the corresponding mucosas of cancer-associated intestinal metaplasias and 3\% of cancer-negative intestinal metaplasias, in which the telomere volume is markedly reduced (35). The status of gastric mucosa was classified according to inflammation and immortalization by $h T E R T$ expression. AKT is a key protein that links inflammation and tumorigenesis. Therefore, AKT became the focus of previous studies (9-13). iNOS is an important mediator of inflammation in the gastric mucosa, and increased expression of $i N O S$ is epigenetically induced upon $H$. pylori infection as a host defense (37). NT is a marker of nitric oxide (NO)-induced protein degradation. In a previous study, the expression of iNOS and $N T$ was examined to evaluate inflammation and the expression of $h T E R T$ as a marker of immortality (13). The data revealed that the levels of pAKT are associated with $h T E R T$ expression in CGA (13). In addition, it was also revealed in this study that pAKT levels are associated with hTERT expression in gastric adenocarcinomas (13). Overall, these findings indicated that the immortality of gastric mucosal cells is associated with inflammation-induced AKT activation.

pAKT levels are elevated in certain types of gastric mucosa, such as CAG, CMG and CGA (13). Notably, high pAKT levels in CAG are associated with $H$. pylori-induced active inflammation. By contrast, the increased pAKT levels in CMG suggest that persistent iNOS upregulation develops during the metaplastic process. The highest pAKT levels in CGA may develop during carcinogenic processes (38). In vitro analysis has revealed that only CGA and MKN28 cancer cells demonstrated upregulation of pAKT in response to brief $\mathrm{NO}$ exposure. In Barrett's esophagus, activation of AKT is associated with dysplasia-carcinoma sequence (39). In addition, 
only CGA has been found to express hTERT (13). These findings suggest that CGA is likely to be a distinct category in the process of carcinogenesis. The activation of telomere shortening induced by $H$. pylori infection leads to hTERT expression and telomerase activation (Fig. 1).

hTERT activity is regulated by phosphorylation, in addition to the expression of $h T E R T$. Protein kinase $\mathrm{C}$ and AKT each phosphorylate hTERT $(40,41)$. AKT-catalyzed phosphorylation of hTERT induces intranuclear translocation of hTERT and subsequently, activates hTERT. By contrast, ring finger protein 1, which is an E3 ubiquitin ligase, decreases the activity of hTERT by ubiquitination (42).

AKT is associated with diverse pro-tumor responses, including hTERT activation (9-13). In a previous study, the significance of AKT phosphorylation and hTERT on the prognosis of gastric cancer was examined (43). The activation of AKT by epidermal growth factor was found to increase hTERT expression and telomerase activity. By contrast, AKT inactivation by inhibitors and knockdown decreased $h T E R T$ expression and telomerase activity in MKN28 gastric cancer cells (43). In 40 gastric cancer tissues, a significant association was found between the levels of pAKT and hTERT expression and telomere length (43). The levels of pAKT or pAKT/hTERT are not associated with clinicopathological parameters, such as the tumor stage and presence of nodal metastasis. However, the survival rate of the patients with a high level of pAKT or the patients with a high level of pAKT and hTERT has been found to be significantly worse compared with other patients (43). These findings suggest that AKT and hTERT are good molecular targets for the treatment of gastric cancer.

AKT is associated with cancer cell survival through the alteration of the expression of $\mathrm{Bcl}-2$ antagonist of cell death, p53, forkhead, nuclear factor $\kappa \mathrm{B}, \mathrm{mTOR}$ and PTEN (10) (44). In addition, dysregulated PTEN/PI3K/AKT signaling interacts with the Wnt signaling pathway to induce epithelial-mesenchymal transition (EMT), which is usually associated with the cancer stem cell phenotype and a poor prognosis (45). A previous study has reported that hTERT promotes EMT induced by transforming growth factor- $\beta$ and $\beta$-catenin by inducing $\beta$-catenin nuclear translocation and its transcriptional activity for vimentin, a mesenchymal factor, expression (46). Therefore, PTEN/PI3K/AKT signaling enhances EMT and stem cell phenotypes. In a previous study, the association between AKT phosphorylation, TERT expression and telomerase activity was confirmed in MKN28 gastric cancer cells and gastric cancer tissues (43). These associations may result in poor prognoses in patients with high pAKT levels or high pAKT/hTERT levels. Furthermore, multivariate analysis has revealed that pAKT and pAKT/hTERT levels are reliable prognostic factors. The examination of additional gastric cancer cases is required to confirm the hypothesis that the EMT/stem cell phenotype affects disease progression (43-46).

Angiogenesis is an essential phenotype for cancer progression, and VEGF expression is associated closely with neovascularization and cancer progression in numerous malignancies (47). The PI3K/AKT pathway induces VEGF response, which includes other downstream inducers, including mitogen-activated protein kinase (extracellular signal-regulated kinases or p38), Src, focal adhesion kinase, Rho family GTPases, and endothelial NO (48). The PI3K/AKT pathway increases the secretion of VEGF from cancer cells by hypoxia-inducible factor 1-dependent and independent mechanisms (49). Therefore, AKT suppression may result in an anti-angiogenic effect on gastric cancer.

This data revealed that AKT and hTERT are present in high levels in gastric cancer, and the concurrent synthesis of these two proteins at high levels is associated with a poor prognosis (43). These results suggest that AKT and hTERT are possible molecular targets for the treatment of gastric cancer.

\section{Conclusion}

In gastric carcinogenesis, H. pylori is an essential factor for the stimulation of transformation in gastric epithelial cells. As shown in Fig. 1, H. pylori induces iNOS, synthesis of ROS and telomere shortening as a result of repetitive destruction/regeneration cycles in chronic active gastritis. AKT plays a central role in $H$. pylori-induced gastric carcinogenesis. H. pylori-induced oxidative stress activates AKT, and $H$. pylori-accelerated cell growth in the gastric mucosa shortens telomere length. These changes lead to hTERT expression and telomerase activation. Telomerase activity immortalizes epithelial cells to enable uncontrolled cancer cell division and instigates malignant phenotypes, therefore resulting in the progression of cancer. These findings demonstrated that AKT plays a pivotal role in gastric cancer development. Thus, the investigation of AKT as a promising molecular target to prevent the development or progression of gastric cancer should be considered.

\section{References}

1. Siegel R, Naishadham D and Jemal A: Cancer statistics, 2012. CA Cancer J Clin 62: 10-29, 2012.

2. Yokozaki H, Kuniyasu H, Semba S, Yasui W and Tahara E: Molecular bases of human stomach carcinogenesis. Mol Pathol Gastroenterol Cancer. Tahara E (ed.) Springer-Verlag, Tokyo, pp55-70, 1997.

3. Kuniyasu H, Yasui W, Yokozaki H and Tahara E: Helicobacter pylori infection and carcinogenesis of the stomach. Langenbecks Arch Surg 385: 69-74, 2000.

4. IARC: Schistosomes, Liver Flukes and Hericobactor pylori. IARC Sci, 1994.

5. Tsukamoto T and Tatematsu M: Role of Helicobacter pylori in gastric neoplasia. Curr Infect Dis Rep 16: 402, 2014.

6. Sukawa Y, Yamamoto $\mathrm{H}$, Nosho K, et al: Alterations in the human epidermal growth factor receptor 2-phosphatidylinositol 3-kinase-v-Akt pathway in gastric cancer. World J Gastroenterol 18: 6577-6586, 2012.

7. Berg M and Soreide K: EGFR and downstream genetic alterations in KRAS/BRAF and PI3K/AKT pathways in colorectal cancer: implications for targeted therapy. Discov Med 14: 207-214, 2012.

8. Ng L, Poon RT and Pang R: Biomarkers for predicting future metastasis of human gastrointestinal tumors. Cell Mol Life Sci 70: 3631-3656, 2013.

9. Cheung M and Testa JR: Diverse mechanisms of AKT pathway activation in human malignancy. Curr Cancer Drug Targets, 2013.

10. Downward J: PI 3-kinase, Akt and cell survival. Semin Cell Dev Biol 15: 177-182, 2004.

11. Radisavljevic Z: AKT as locus of cancer angiogenic robustness and fragility. J Cell Physiol 228: 21-24, 2013.

12. Xu M and Mo YY: The Akt-associated microRNAs. Cell Mol Life Sci 69: 3601-3612, 2012.

13. Sasaki T, Kuniyasu H, Luo Y, et al: Increased phosphorylation of AKT in high-risk gastric mucosa. Anticancer Res 33: 3295-3300, 2013.

14. Ho SB: Premalignant lesions of the stomach. Semin Gastrointest Dis 7: 61-73, 1996. 
15. Hatakeyama M: Oncogenic mechanisms of the Helicobacter pylor CagA protein. Nat Rev Cancer 4: 688-694, 2004.

16. Higashi H, Tsutsumi R, Muto S, et al: SHP-2 tyrosine phosphatase as an intracellular target of Helicobacter pylori CagA protein. Science 295: 683-686, 2002.

17. You M, Yu DH and Feng GS: Shp-2 tyrosine phosphatase functions as a negative regulator of the interferon-stimulated Jak/STAT pathway. Mol Cell Biol 19: 2416-2424, 1999.

18. Tosh D and Slack JM: How cells change their phenotype. Nat Rev Mol Cell Biol 3: 187-194, 2002.

19. Ochiai A and Hirohashi S: Genetic alteration in the precursors of gastric cancer. Mol Pathol Gastroenterol Cancer. Tahara E (ed.) Springer-Verlag, Tokyo pp43-53, 1997.

20. Sugano K: Premalignant conditions of gastric cancer J Gastroenterol Hepatol 28: 906-911, 2013.

21. Testino G: Helicobacter pylori, cell proliferation and carcinogenesis. Am J Gastroenterol 96: 2514, 2001.

22. Guerci A, Chambre JF, Franck P, Floquet J, Gaucher P and Guerci O: Flow cytometric analysis of the cell cycle in chronic gastritis. Anal Cell Pathol 4: 381-388, 1992.

23. Tahara E, Kuniyasu H, Yasui W and Yokozaki H: Gene alterations in intestinal metaplasia and gastric cancer. Eur J Gastroenterol Hepatol (6 Suppl 1): 102, 1994.

24. Ochiai A, Yamauchi Y and Hirohashi S: p53 mutations in the non-neoplastic mucosa of the human stomach showing intestina metaplasia. Int J Cancer 69: 28-33, 1996.

25. Semba S, Yokozaki H, Yamamoto S, Yasui W and Tahara E: Microsatellite instability in precancerous lesions and adenocarcinomas of the stomach. Cancer 77: 1620-1627, 1996.

26. Blackburn EH: Switching and signaling at the telomere. Cell 106 661-673, 2001.

27. Lingner J, Cooper JP and Cech TR: Telomerase and DNA end replication: no longer a lagging strand problem? Science 269: $1533-1534,1995$.

28. Stewart SA and Weinberg RA: Telomeres: cancer to human aging. Annu Rev Cell Dev Biol 22: 531-557, 2006.

29. Tahara H, Kuniyasu H, Yokozaki H, et al: Telomerase activity in preneoplastic and neoplastic gastric and colorectal lesions. Clin Cancer Res 1: 1245-1251, 1995 .

30. Feng J, Funk WD, Wang SS, et al: The RNA component of human telomerase. Science 269: 1236-1241, 1995.

31. Avilion AA, Piatyszek MA, Gupta J, Shay JW, Bacchetti S and Greider CW: Human telomerase RNA and telomerase activity in immortal cell lines and tumor tissues. Cancer Res 56: 645-650, 1996.

32. Blasco MA, Rizen M, Greider CW and Hanahan D: Differential regulation of telomerase activity and telomerase RNA during multi-stage tumorigenesis. Nat Genet 12: 200-204, 1996.

33. Kuniyasu H, Domen T, Hamamoto T, et al: Expression of human telomerase RNA is an early event of stomach carcinogenesis. Jpn J Cancer Res 88: 103-107, 1997.
34. Weiss H, Gutz HJ, Schroter J and Wildner GP: DNA distribution pattern in chronic gastritis. I. DNA ploidy and cell cycle distribution. Scand J Gastroenterol 24: 643-648, 1989.

35. Kuniyasu H, Kitadai Y, Mieno H and Yasui W: Helicobactor pylori infection is closely associated with telomere reduction in gastric mucosa. Oncology 65: 275-282, 2003.

36. Autexier $C$ and Lue NF: The structure and function of telomerase reverse transcriptase. Annu Rev Biochem 75: 493-517, 2006

37. Angrisano T, Lembo F, Peluso S, Keller S, Chiariotti L and Pero R: Helicobacter pylori regulates iNOS promoter by histone modifications in human gastric epithelial cells. Med Microbiol Immunol 201: 249-257, 2012.

38. Murata M, Thanan R, Ma N and Kawanishi S: Role of nitrative and oxidative DNA damage in inflammation-related carcinogenesis. J Biomed Biotechnol 2012: 623019, 2012.

39. Beales IL, Ogunwobi O, Cameron E, El-Amin K, Mutungi G and Wilkinson $\mathrm{M}$ : Activation of Akt is increased in the dysplasia-carcinoma sequence in Barrett's oesophagus and contributes to increased proliferation and inhibition of apoptosis: a histopathological and functional study. BMC Cancer 7: 97, 2007.

40. Li H, Zhao L, Yang Z, Funder JW and Liu JP: Telomerase is controlled by protein kinase $\mathrm{C}$ alpha in human breast cancer cells. J Biol Chem 273: 33436-33442, 1998.

41. Kang SS, Kwon T, Kwon DY and Do SI: Akt protein kinase enhances human telomerase activity through phosphorylation of telomerase reverse transcriptase subunit. J Biol Chem 274: 13085-13090, 1999

42. Kim JH, Park SM, Kang MR, et al: Ubiquitin ligase MKRN1 modulates telomere length homeostasis through a proteolysis of hTERT. Genes Dev 19: 776-781, 2005.

43. Sasaki T, Kuniyasu H, Luo Y, et al: AKT activation and telomerase reverse transcriptase expression are concurrently associated with prognosis of gastric cancer. Pathobiology 81: 36-41, 2014.

44. Populo H, Lopes JM and Soares P: The mTOR Signalling Pathway in Human Cancer. Int J Mol Sci 13: 1886-1918, 2012.

45. Karamitopoulou E: Tumor budding cells, cancer stem cells and epithelial-mesenchymal transition-type cells in pancreatic cancer. Front Oncol 2: 209, 2012

46. Liu Z, Li Q, Li K, et al: Telomerase reverse transcriptase promotes epithelial-mesenchymal transition and stem cell-like traits in cancer cells. Oncogene 32: 4203-4213, 2012.

47. Fidler IJ: The pathogenesis of cancer metastasis: the 'seed and soil' hypothesis revisited. Nat Rev Cancer 3: 453-458, 2003.

48. Claesson-Welsh L and Welsh M: VEGFA and tumour angiogenesis. J Intern Med 273: 114-127, 2013.

49. Karar J and Maity A: PI3K/AKT/mTOR Pathway in Angiogenesis. Front Mol Neurosci 4: 51, 2011. 